\title{
Molecules in the circumstellar disk orbiting BP Piscium (Research Note)
}

\author{
J. H. Kastner ${ }^{1,2}$, B. Zuckerman ${ }^{3,4}$, and T. Forveille ${ }^{1}$ \\ ${ }^{1}$ Laboratoire d'Astrophysique de Grenoble, Université Joseph Fourier - CNRS, BP 53, 38041 Grenoble Cedex, France \\ e-mail: joel.kastner@obs.ujf-grenoble.fr \\ 2 Chester F. Carlson Center for Imaging Science, Rochester Institute of Technology, 54 Lomb Memorial Dr., Rochester, NY 14623, \\ USA \\ 3 Dept. of Physics \& Astronomy, University of California, Los Angeles 90095, USA \\ 4 UCLA Center for Astrobiology, University of California, Los Angeles 90095, USA
}

Received 7 March 2008 / Accepted 10 May 2008

\section{ABSTRACT}

\begin{abstract}
Context. BP Psc is a puzzling late-type, emission-line field star with large infrared excess. The star is encircled and enshrouded by a nearly edge-on, dusty circumstellar disk, and displays an extensive jet system similar to those associated with pre-main sequence (pre-MS) stars. However, the photospheric absorption features of the star itself appear more consistent with post-main sequence status. Aims. We seek to characterize the molecular gas component of the BP Psc disk, so as to compare the properties of its molecular disk with those of well-studied pre-main sequence stars.

Methods. We conducted a mm-wave molecular line survey of BP Psc with the $30 \mathrm{~m}$ telescope of the Institut de Radio Astronomie Millimetrique (IRAM). We use these data to investigate the kinematics, gas mass, and chemical constituents of the BP Psc disk.

Results. We detected lines of ${ }^{12} \mathrm{CO}$ and ${ }^{13} \mathrm{CO}$ and, possibly, very weak emission from $\mathrm{HCO}^{+}$and $\mathrm{CN} ; \mathrm{HCN}, \mathrm{H}_{2} \mathrm{CO}$, and $\mathrm{SiO}$ are not detected. The CO line profiles of BP Psc are well fit by a model invoking a disk in Keplerian rotation. The mimumum disk gas mass, inferred from the ${ }^{12} \mathrm{CO}$ line intensity and ${ }^{13} \mathrm{CO} /{ }^{12} \mathrm{CO}$ line ratio, is $\sim 0.1$ Jupiter masses.

Conclusions. The weakness of $\mathrm{HCO}^{+}$and $\mathrm{CN}$ (relative to ${ }^{13} \mathrm{CO}$ ) stands in sharp contrast to the strong $\mathrm{HCO}^{+}$and $\mathrm{CN}$ emission that characterizes most low-mass, pre-main sequence stars that have been the subjects of molecular emission-line surveys, and is suggestive of a very low level of X-ray-induced molecular ionization within the BP Psc disk. These results lend some support to the notion that BP Psc is an evolved star whose circumstellar disk has its origins in a catastrophic interaction with a close companion.
\end{abstract}

Key words. stars: circumstellar matter - stars: individual: BP Psc - planetary systems: protoplanetary disks - ISM: molecules

\section{Introduction}

Circumstellar disks around young stars serve both as the sources of material for accreting young stars and as the sites of nascient planets orbiting such stars. Circumstellar disks around main sequence and evolved stars may represent debris resulting from planetary collisions, the destruction of planetary-mass companions, or similarly devastating interactions with stellar-mass companions. Studies of such disks typically rely on dust emission (i.e., infrared excess) to ascertain fundamental disk properties (e.g., disk dimensions and mass; e.g., Backman \& Paresce 1993; Lagrange et al. 2000; Zuckerman 2001). Complementary, sensitive measurements of (sub)millimeter emission from $\mathrm{CO}$ (after $\mathrm{H}_{2}$, the second-most abundant molecular species) as well as less abundant molecular species (such as $\mathrm{HCN}, \mathrm{CN}$, and $\mathrm{HCO}^{+}$) toward disk-enshrouded stars provide the best available means to determine the residual molecular gas content and chemistry of circumstellar disks (e.g., Zuckerman et al. 1995; Kastner et al. 1997; Dutrey et al. 1997; Thi et al. 2004). Such measurements provide unique tests for theories describing disk chemical and thermal evolution, particularly for those models concerned with the effects of irradiation of protoplanetary disks by high-energy photons - UV and (perhaps more importantly) X-rays - on circumstellar disk chemistry and energetics (e.g., Glassgold et al. 2004, 2007).
The enigmatic $\mathrm{H} \alpha$ emission-line field star BP Psc (=StH $\alpha$ 202; Stephenson 1986) stands as an important object in this regard (Zuckerman et al. 2008, hereafter Z08). Z08 established that this little-studied system consists of a late G-type or early K-type star surrounded by a compact, dusty, gaseous disk, and that the star-disk system is the source of highly collimated jets. Z08 furthermore infer, on the basis of both its classical, double-peaked CO line profiles and the dark-lane morphology of near-IR adaptive optics images, that the BP Psc disk is viewed nearly edge-on $\left(i \sim 75^{\circ}\right)$ and that the disk absorbs and reradiates $75 \%$ of the incident stellar luminosity as seen from Earth.

Like the intensively-studied, nearby classical $\mathrm{T}$ Tauri star (cTTS) TW Hya (Kastner et al. 1997; Webb et al. 1999, and references therein), BP Psc is isolated and found at high galactic latitude. Z08 show that if BP Psc is an early K-type premain sequence (pre-MS) star then, like TW Hya, it would be one of the closest (and perhaps oldest) classical T Tauri stars known. However, unlike TW Hya, no young stellar association has been identified in the vicinity of BP Psc (for a list of nearby $[D \lesssim 100 \mathrm{pc}]$ young associations, see review in Zuckerman \& Song 2004). Indeed, Z08 also present two lines of evidence suggesting that BP Psc may be an evolved star-most likely, a firstascent giant: its $\lambda 6709.6 \mathrm{Li}$ absorption line is far weaker than in early K-type stars of age <100 Myr, and gravity-sensitive lines in 
Table 1. Molecular emission lines measured toward BP Psc.

\begin{tabular}{|c|c|c|c|c|c|c|}
\hline $\begin{array}{l}\text { Transition } \\
\text { (1) }\end{array}$ & $\begin{array}{c}v \\
(\mathrm{GHz}) \\
(2)\end{array}$ & $\begin{array}{c}T_{B, \max } \\
(\mathrm{mK}) \\
(3)\end{array}$ & $\begin{array}{c}I \\
\left(\mathrm{~K} \mathrm{~km} \mathrm{~s}^{-1}\right) \\
(4)\end{array}$ & $\begin{array}{c}v_{\mathrm{d}} \\
\left(\mathrm{km} \mathrm{s}^{-1}\right) \\
(5)\end{array}$ & $\begin{array}{l}q \\
(6)\end{array}$ & $\begin{array}{l}p_{\mathrm{d}} \\
\text { (7) }\end{array}$ \\
\hline${ }^{12} \mathrm{CO}(1-0)$ & 115.270 & $85(4)$ & $1.2(0.1)$ & $5.3(0.3)$ & $0.8(0.1)$ & 0.33 : \\
\hline${ }^{12} \mathrm{CO}(2-1)$ & 230.538 & $260(35)$ & $3.5(0.4)$ & $4.7(0.3)$ & $0.7(0.1)$ & $0.1:$ \\
\hline${ }^{12} \mathrm{CO}(3-2)$ & 345.796 & $530(120)$ & $5.2(0.8)$ & $4.0(0.5)$ & 0.5 : & 0.1: \\
\hline${ }^{13} \mathrm{CO}(2-1)$ & 220.399 & $33(2)$ & $0.4(0.1)$ & $4.0(0.9)$ & $0.9(0.2)$ & $\ldots$ \\
\hline $\mathrm{HCO}^{+}(3-2)$ & 267.558 & $<15$ & $<0.23$ & $\ldots$ & $\ldots$ & $\ldots$ \\
\hline $\mathrm{CN}(2-1)$ & 226.875 & $<9$ & $<0.15$ & $\ldots$ & $\ldots$ & $\ldots$ \\
\hline $\mathrm{SiO}(v=1,2-1)$ & 86.243 & $<12$ & $<0.18$ & $\ldots$ & $\ldots$ & $\ldots$ \\
\hline HCN (1-0) & 88.632 & $<4$ & $<0.06$ & $\ldots$ & $\ldots$ & $\ldots$ \\
\hline $\mathrm{HCN}(3-2)$ & 265.886 & $<70$ & $<1.1$ & $\ldots$ & $\ldots$ & $\ldots$ \\
\hline $\mathrm{H}_{2} \mathrm{CO}\left(2_{21}-1_{11}\right)$ & 140.839 & $<10$ & $<0.15$ & $\ldots$ & $\ldots$ & $\ldots$ \\
\hline
\end{tabular}

Notes - Data obtained in 2007 December with the IRAM 30 m, with exception of ${ }^{12} \mathrm{CO}(2-1)$ (obtained 1996 May with the IRAM 30 m) and ${ }^{12} \mathrm{CO}(3-2)$ (obtained 1996 Feb. with the JCMT 15 m). Peak line intensity $\left(T_{B, \max }\right)$, integrated line intensity $(I)$, one-half peak-to-peak velocity difference $\left(v_{\mathrm{d}}\right)$, radial temperature power law index $(q)$, and outer disk cutoff parameter $\left(p_{\mathrm{d}}\right)$ obtained from fits to disk model line profiles. Numbers in parentheses indicate formal $(1 \sigma)$ uncertainties in best-fit parameter values. Upper limits on $T_{B, \max }$ and $I$ listed for nondetected transitions of $\mathrm{SiO}, \mathrm{HCN}$, and $\mathrm{H}_{2} \mathrm{CO}$ are $3 \sigma$, based on measured channel-to-channel noise levels in off-line regions of spectrum and an assumed linewidth of $15 \mathrm{~km} \mathrm{~s}^{-1}$. See Sects. 2.2 and 3.1 .

its optical spectrum suggest it is of luminosity class IV or III, i.e., its surface gravity is lower than a typical 10 Myr-old, K-type, pre-MS star. Whether BP Psc is an isolated, relatively old cTTS or a post-MS star undergoing an episode of collimated mass loss, its nearly edge-on disk and its system of jets and Herbig-Haro objects - which is as spectacular as the jet systems commonly associated with very young (still cloud-embedded) pre-MS stars - make BP Psc an exceedingly unusual object.

To better characterize the disk orbiting BP Psc, we undertook a mm-wave molecular line survey with the $30 \mathrm{~m}$ telescope of the Institut de Radio Astronomie Millimetrique (IRAM ${ }^{1}$ ). In addition to constraining the mass, kinematics, and chemistry of the BP Psc disk, the results point out significant differences between this system and those pre-MS star molecular disks that have also been the subjects of extensive radio emission-line surveys.

\section{Observations}

\subsection{Data acquisition and reduction}

We conducted our molecular line survey of BP Psc with the IRAM $30 \mathrm{~m}$ telescope during the period 4-6 Dec. 2007. The molecules and transitions observed are listed in Table 1 . We observed simultaneously in either the $100 \mathrm{GHz}(3 \mathrm{~mm})$ and $230 \mathrm{GHz}(1 \mathrm{~mm})$ or $150 \mathrm{GHz}(2 \mathrm{~mm})$ and $270 \mathrm{GHz}(1 \mathrm{~mm})$ bands, and in both polarizations in each band, using receiver combinations $\mathrm{A} 100+\mathrm{B} 100$ and $\mathrm{C} 230+\mathrm{D} 230$ or $\mathrm{A} 150+\mathrm{B} 150$ and C270+D270 (all in SSB mode), respectively. The $1 \mathrm{MHz}$ filter banks served as the spectral line backends. The weather was excellent to good $\left(\tau_{225} \sim 0.1\right.$ to 0.3$)$ throughout the period; time-averaged system temperatures in both the $3 \mathrm{~mm}$ and $1 \mathrm{~mm}$ bands were in the range $250-400 \mathrm{~K}$. We checked pointing and focus (using Uranus as the reference) every $1-2 \mathrm{~h}$, and both were found to be stable and reliable; typical pointing errors were $<3^{\prime \prime}$, i.e., less than $1 / 8$ beamwidth for $3 \mathrm{~mm}$ (FWHP beamwidth $21^{\prime \prime}$ ) and less than $1 / 4$ beamwidth for $1 \mathrm{~mm}$ (FWHP beamwidth $12^{\prime \prime}$ ). Individual spectral scans were of duration $200 \mathrm{~s}$, with total integration times (per polarization) ranging from $\sim 40 \mathrm{~min}$ (for $\mathrm{HCN}(3-2)$ ) to $\sim 8.5 \mathrm{~h}$ (for $\mathrm{HCO}^{+}(3-2)$ ).

1 http://iram.fr/
We used the CLASS ${ }^{2}$ radio spectral line data reduction package to sum all individual spectral scans obtained in both polarizations for a given transition, and then to subtract a linearfit baseline from each of these integrated spectra, calculating channel-to-channel noise levels in the process. A few individual scans were discarded due to baseline anomalies. All antenna temperature measurements reported in Table 1 (i.e., $T_{B, \max }$ and $I$; see Sect. 3.1), have been corrected for beam efficiency assuming $B_{\text {eff }}=0.76,0.70,0.57$ and 0.45 at observing frequencies of 86 , 115,230 , and $270 \mathrm{GHz}$, respectively ${ }^{3}$.

\subsection{Results}

Results are summarized in Table 1 and Fig. 1. Including the previous observations obtained with the IRAM $30 \mathrm{~m}$ and the $15 \mathrm{~m}$ James Clerk Maxwell Telescope (JCMT) reported in Z08, the BP Psc molecular line survey has yielded detections of ${ }^{12} \mathrm{CO}(1-0),{ }^{12} \mathrm{CO}(2-1),{ }^{12} \mathrm{CO}(3-2)$ and ${ }^{13} \mathrm{CO}(2-1)$ emission; only tentative detections of $\mathrm{HCO}^{+}(3-2)$ and $\mathrm{CN}(2-1)$ emission; and nondetections of $\mathrm{HCN}, \mathrm{H}_{2} \mathrm{CO}$, and $\mathrm{SiO}$ (maser) emission. Given the marginal $(\sim 2 \sigma)$ significance of the possible detections of the $\mathrm{HCO}^{+}(3-2)$ and $\mathrm{CN}(2-1)$ lines, these observations are reported as upper limits in Table 1 and are considered as such in the discussion below. For $\mathrm{HCN}, \mathrm{H}_{2} \mathrm{CO}$, and $\mathrm{SiO}$, the upper limits on peak antenna temperature $T_{B \text {,max }}$ and integrated line intensity $I$ listed in Table 1 were obtained from the channelto-channel noise level measured via spectral baseline fitting, assuming a linewidth of $15 \mathrm{~km} \mathrm{~s}^{-1}$ FWHM (as estimated from CO line profile fitting; Sect. 3.1). The measurements of the $\mathrm{CO}$ line profile parameters and of the upper limits on $T_{B, \max }$ and $I$ for the marginal detections of $\mathrm{HCO}^{+}$and $\mathrm{CN}$ are described in Sect. 3.1. Relative to the intensities of the $\mathrm{CO}$ lines, the $\mathrm{HCO}^{+}, \mathrm{HCN}(1-0)$, and $\mathrm{CN}$ emission from the BP Psc disk is evidently quite weak in comparison to pre-MS star disks (Dutrey et al. 1997; Thi et al. 2004, see Sect. 3.3). The upper limit on $\operatorname{HCN}(3-2)$ emission in Table 1 is not similarly constraining.

\footnotetext{
${ }^{2}$ See http://iram.fr/IRAMFR/GILDAS/

3 See http://iram.fr/IRAMFR/ARN/aug05/node6.html
} 


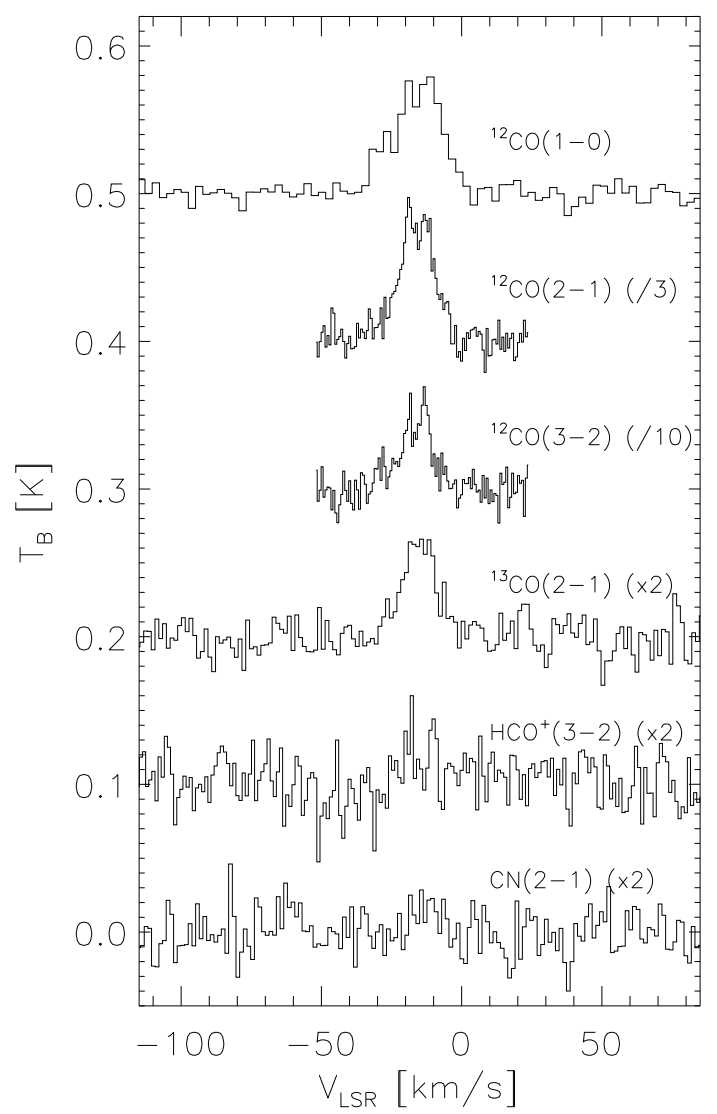

Fig. 1. Radio (mm-wave) molecular spectra of BP Psc. Ordinate is velocity with respect to the Local Standard of Rest (LSR) and abscissa is antenna temperature corrected for beam efficiency. Spectral baselines are offset in $T_{B}$ for clarity. All spectra except ${ }^{12} \mathrm{CO}(3-2)$ were obtained with the IRAM $30 \mathrm{~m} ;{ }^{12} \mathrm{CO}(3-2)$ was obtained at the JCMT $15 \mathrm{~m}$. See text and Table 1.

\section{Analysis and discussion}

\subsection{CO line profiles: constraints on disk structure and kinematics}

Some of the CO lines displayed in Fig. 1 (see also Z08) appear to display the double-peaked profile characteristic of a circumstellar disk in Keplerian rotation (e.g., Beckwith \& Sargent 1993, hereafter BS93, Omodaka et al. 1992). In contrast, the ${ }^{13} \mathrm{CO}(2-$ 1) line appears flat-topped, and is reminiscent of the ${ }^{12} \mathrm{CO}(3-2)$ line profile measured with the SubMillimeter Array (Z08). All of the CO lines display broad wings extending $\pm \sim 15 \mathrm{~km} \mathrm{~s}^{-1}$ to either side of the systemic velocity of BP Psc $\left(V_{\mathrm{LSR}}=\right.$ $\left.-15 \mathrm{~km} \mathrm{~s}^{-1}\right)$. In addition, the ${ }^{12} \mathrm{CO}(1-0)$ line appears to display a broad negative-velocity shoulder; a feature near $V_{\mathrm{LSR}} \sim$ $-25 \mathrm{~km} \mathrm{~s}^{-1}$ may be present in the other $\mathrm{CO}$ profiles as well.

The CO line profile asymmetries, and the slight variation from transition to transition, suggests departures from the "ideal" flattened disk with sharp outer edge in Keplerian rotation. Nevertheless, the BP Psc CO profiles can be reasonably well described by a simple parameterization of the results of detailed numerical models of Keplerian molecular disks surrounding T Tauri stars (Eqs. (27), (28) of BS93). The parameterization applies in the case of a disk viewed edge-on - a reasonable approximation of the BP Psc viewing geometry - and is in terms of the Keplerian velocity $v_{\mathrm{d}}$ at the outer edge of the disk $\left(v_{\mathrm{d}}=\left(G M_{\star} / R_{\mathrm{d}}\right)^{1 / 2}\right.$, where $M_{\star}$ is the stellar mass and $R_{\mathrm{d}}$ the disk outer radius) and the index $q$ of the (assumed) power law dependence of disk gas temperature $T$ on radial position $r$ (i.e., $\left.T(r) \propto r^{-q}\right)$. Hence, $v_{\mathrm{d}}$ defines the peaks of the "twin horns" in the ideal line profile, with the value of $v_{\mathrm{d}}$ roughly equal to half the peak-to-peak velocity separation of the horns. Because the velocity dependence of line flux is $F(v) \propto v^{3 q-5}$ for $v>v_{\mathrm{d}}$ (BS93), $q$ dictates the steepness of the line wings in the model profiles. Values of $q$ larger than the canonical $q=1 / 2$ yield an "excess" of high- $v$ disk material relative to the "standard" BS93 disk models and, hence, broader wings than those of the BS93 profiles. In modeling the BP Psc CO line profiles, we modified the low- $v$ BS93 profile parameterization (BS93, Eq. (28)) by introducing a variable index for the power-law dependence of the line intensity on velocity (i.e., $F(v) \propto v^{p_{\mathrm{d}}}$ for $v<v_{\mathrm{d}}$ ). This parameter, $p_{\mathrm{d}}$, in effect accounts for the fact that the disk likely does not have a sharp cutoff at $R_{\mathrm{d}}$; values of $p_{\mathrm{d}}<1.0$ tend to fill in the central regions of the line profile.

Fitting the BP Psc CO lines with this simple model thereby allows the empirical determination of $v_{\mathrm{d}}$ and the peak line intensity $T_{B, \max }$, as well as the temperature profile and outer edge cutoff power-law indices $q$ and $p_{\mathrm{d}}$. For the three ${ }^{12} \mathrm{CO}$ lines, all four parameters were left free during the model fitting. For the (lower signal-to-noise) ${ }^{13} \mathrm{CO}(2-1)$ line, the value of $p_{\mathrm{d}}$ was fixed to the value estimated from the ${ }^{12} \mathrm{CO}(2-1)$ profile fitting (see below). The total CO line intensities $(I)$ were then obtained by integrating the best-fit models over the velocity range -15 to $+15 \mathrm{~km} \mathrm{~s}^{-1}$ with respect to the systemic velocity of BP Psc. For the (marginal significance) $\mathrm{CN}$ and $\mathrm{HCO}^{+}$lines, all parameters except $T_{B \text {,max }}$ were fixed to the values obtained from the fit to the ${ }^{12} \mathrm{CO}(2-1)$ profile, such that $T_{B \text {, max }}$ was left as the only free parameter; the $3 \sigma$ upper limits on $T_{B \text {, max }}$ and $I$ in Table 1 are then based on the formal uncertainties in the resulting fit to $T_{B, \max }$ (these upper limits are similar to those estimated from the baseline fitting procedure).

The results of this profile fitting exercise are listed in Cols. 3-7 of Table 1. In Fig. 2 we display the best-fit model $\mathrm{CO}$ profiles overlaid on the IRAM and JCMT spectra. For three of the four $\mathrm{CO}$ lines measured thus far, we obtain best-fit values of $v_{\mathrm{d}}$ in the range $4.0-4.7 \mathrm{~km} \mathrm{~s}^{-1}$. The ${ }^{12} \mathrm{CO}(1-0)$ line yields a somewhat larger best-fit value of $v_{\mathrm{d}}=5.3 \pm 0.3 \mathrm{~km} \mathrm{~s}^{-1}$. These values of $v_{\mathrm{d}}$ are all systematically larger than those obtained from simple, two-Gaussian model fits to the single-dish (Fig. 1) and interferometer (Z08) CO profiles, which yield peak-to-peak separations in the range $6.0-7.5 \mathrm{~km} \mathrm{~s}^{-1}$ (i.e., $v_{\mathrm{d}}$ in the range $3.0-$ $3.75 \mathrm{~km} \mathrm{~s}^{-1}$ ). The discrepancy is due to the fact that, in the BS93 model parameterization, the value of $v_{\mathrm{d}}$ corresponds to the outer edges of the "twin peaks" in the line profile, whereas the Gaussian fits find the velocity centroids of these peaks.

We (tentatively) conclude that $v_{\mathrm{d}}$ lies in the range 3.0$4.0 \mathrm{~km} \mathrm{~s}^{-1}$. For such a range of $v_{\mathrm{d}}$, the mass of the central star $M_{\star}$ would lie in the range $0.5-0.9(1.0-1.8) M_{\odot}$, given a distance of 100 (300) pc (Z08). The Keplerian profile fits also indicate values of $q$ and $p_{\mathrm{d}}$ in the ranges $0.7-0.9$ and $0.1-0.3$, respectively (Table 1), suggesting that the gas temperature in the BP Psc disk falls off somewhat more steeply than $r^{-1 / 2}$ and that the disk does not have a sharp outer edge.

\subsection{Disk gas mass, density, and gas-to-dust ratio}

Due to lack of knowledge of the optical depth in ${ }^{12} \mathrm{CO}(2-1)$, Z08 were only able to estimate an approximate lower limit to the mass of gas in the BP Psc disk. Detection of ${ }^{13} \mathrm{CO}(2-1)$ emission (Fig. 1, Table 1), in combination with our previous measurement of ${ }^{12} \mathrm{CO}(2-1)$ at the $30 \mathrm{~m}$ (Z08), allows us to refine this disk gas mass estimate. Specifically, the ${ }^{12} \mathrm{CO}(2-1) /{ }^{13} \mathrm{CO}(2-1)$ line ratio 

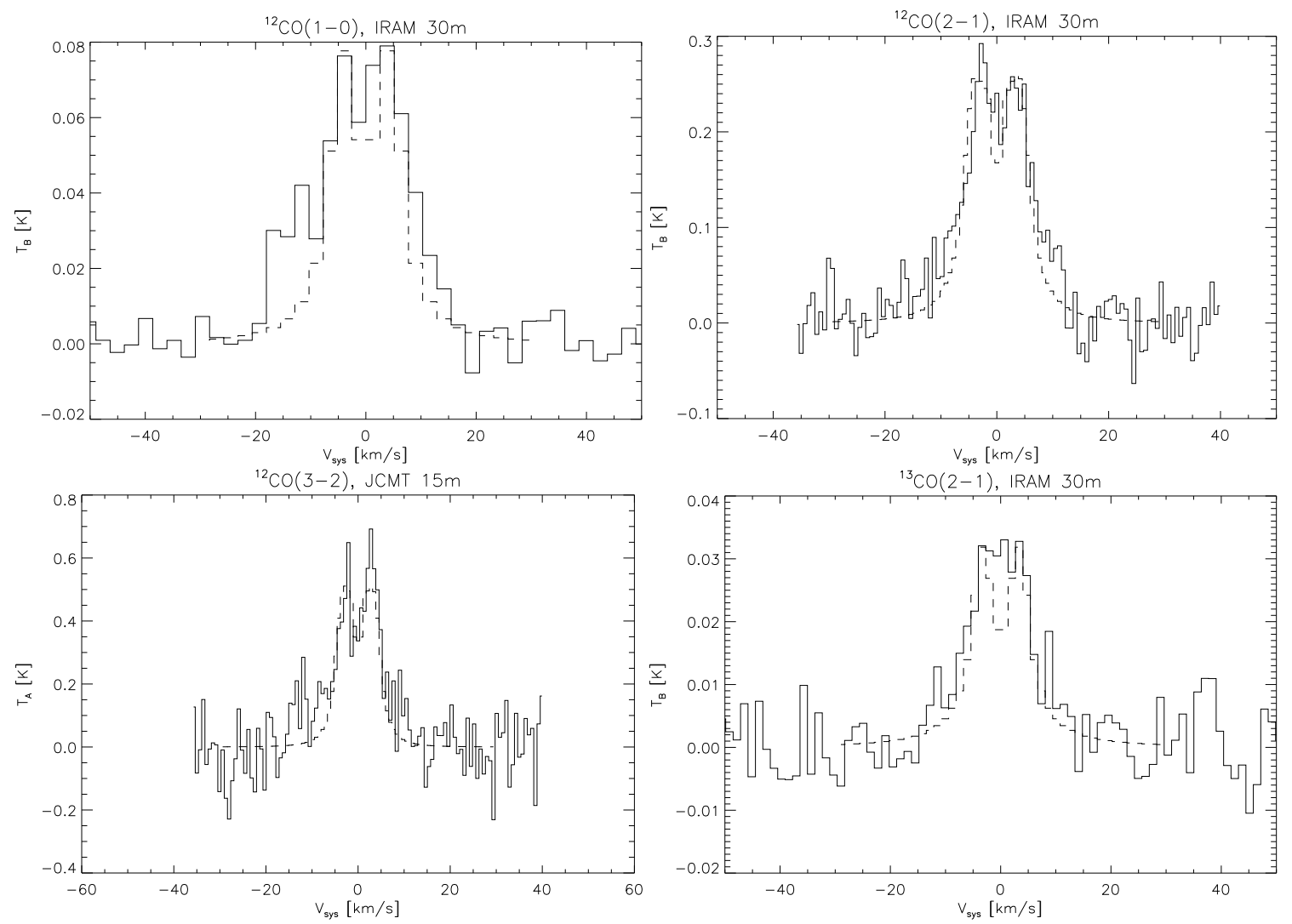

Fig. 2. CO line profiles of BP Psc (solid lines) overlaid with best-fit Keplerian disk model profiles (dashed lines). Ordinate is velocity with respect to the systemic velocity of BP Psc $\left(V_{\mathrm{LSR}}=-15 \mathrm{~km} \mathrm{~s}^{-1}\right)$.

is now determined to be $9 \pm 2$ (Table 1). Assuming the solar value of 89 for the number ratio of ${ }^{12} \mathrm{C}:{ }^{13} \mathrm{C}$, and that the ${ }^{13} \mathrm{CO}(2-$ 1) emission is optically thin (see below), this implies an optical depth in the ${ }^{12} \mathrm{CO}(2-1)$ line of $\tau \sim 10$. With this $\tau$ estimate, and adopting standard formalism (e.g., Eq. (4) in Z08) and standard assumptions (i.e., a mean gas temperature of $\sim 40 \mathrm{~K}$ and a CO: $\mathrm{H}_{2}$ number ratio of $10^{-4}$ ), we estimate a disk gas mass of $\sim 10^{-4} M_{\odot}$ ( $\sim 0.1$ Jupiter masses) assuming that BP Psc is a pre-MS star at a distance of $\sim 100 \mathrm{pc}$. The disk would contain about a Jupiter mass of gas if BP Psc were instead a first-ascent giant at $300 \mathrm{pc}$.

Adopting the Z08 estimate for the mass of cold $(T<$ $200 \mathrm{~K}$ ) dust in the disk, 0.7 Earth masses (for an assumed distance of $100 \mathrm{pc}$ ), these gas mass estimates imply a (distanceindependent) gas-to-dust ratio of $\sim 50$. Meanwhile, assuming BP Psc is pre-MS, such that its disk outer radius is $R_{\mathrm{d}}=50 \mathrm{AU}$ and disk scale height is $5 \mathrm{AU}$ (the latter based on the heavy obscuration of the central star for an assumed inclination $\sim 75^{\circ}$; Z08), the implied mean $\mathrm{H}_{2}$ number density is $n_{\mathrm{H}_{2}} \sim 2 \times$ $10^{8} \mathrm{~cm}^{-3}$. This mean density - which depends only weakly on the assumed distance to, hence evolutionary status of, BP Psc is significantly larger than the critical densities of excitation of all of the molecular transitions in Table 1 (see, e.g., Table 1 of Dutrey et al. 1997).

Our disk gas mass, gas-to-dust ratio, and mean $\mathrm{H}_{2}$ number density estimates remain uncertain - and may still only represent lower limits - given the possibility that most $\mathrm{CO}$ molecules in the disk are frozen out onto (i.e., trapped in icy mantles surrounding) dust grains, or are preferentially photodissociated relative to $\mathrm{H}_{2}$. Indeed, the assumptions invoked above for the $\mathrm{BP}$ Psc disk - optically thin ${ }^{13} \mathrm{CO}$ emission and $\mathrm{CO}: \mathrm{H}_{2}$ number ratio of $10^{-4}-$ may be mutually exclusive. Adopting the standard ISM gas-to-dust ratio of 100, Thi et al. (2004) and
Dutrey et al. (1997) estimate that, in pre-MS disks, CO gas is depleted by factors $\sim 10-200$. Thi et al. (2004) further estimate ${ }^{13} \mathrm{CO}$ optical depths $<1$ for each of the 4 pre-MS disks that they observed with the JCMT, whereas the BS93 CO line profile models indicate that the ${ }^{13} \mathrm{CO}$ optical depth should be $\gg 1$ given a gas-to-dust mass ratio of 100 and $\mathrm{CO}: \mathrm{H}_{2}=10^{-4}$ by number.

\subsection{Weakness of $\mathrm{HCO}^{+}$and $\mathrm{CN}$ : inefficient molecular ionization?}

Models indicate that both $\mathrm{HCO}^{+}$and $\mathrm{CN}$ should be sensitive tracers of X-ray molecular ionization rate at a given gas column density. Glassgold et al. (2004) note that the abundance of $\mathrm{HCO}^{+}$is likely to be sharply elevated in pre-MS circumstellar disks that are irradiated by X-rays from the vicinity of the central star. Although the Glassgold et al. disk models do not include $\mathrm{HCN}$ or $\mathrm{CN}$, the abundance of the latter (the dissociation product of $\mathrm{HCN}$ ) should also be enhanced by exposure to stellar X-rays (Lepp \& Dalgarno 1996). Kastner et al. (1997) and Thi et al. (2004) considered both X-rays and UV as potential drivers of large $\mathrm{HCO}^{+} / \mathrm{CO}$ and $\mathrm{CN} / \mathrm{HCN}$ abundance ratios (relative to the molecular cloud values of these ratios) measured in T Tauri and $\mathrm{Ae} / \mathrm{Be}$ star disks. Ionization of $\mathrm{H}_{2}$ by soft $(\sim 1 \mathrm{keV}) \mathrm{X}$-rays was also implicated as the source of enhanced $\mathrm{HCO}^{+}$in the molecular envelope surrounding the planetary nebula NGC 7027 (Deguchi et al. 1990), an interpretation supported by the subsequent detection of an extended region of luminous, soft X-ray emission within this object (Kastner et al. 2001).

In Figs. 3, 4 we plot the Table 1 results for ${ }^{13} \mathrm{CO} /{ }^{12} \mathrm{CO}$, $\mathrm{HCO}^{+} /{ }^{13} \mathrm{CO}$, and $\mathrm{CN} /{ }^{13} \mathrm{CO}$ line ratios measured for BP Psc along with the same ratios for all (6) other circumstellar disk 


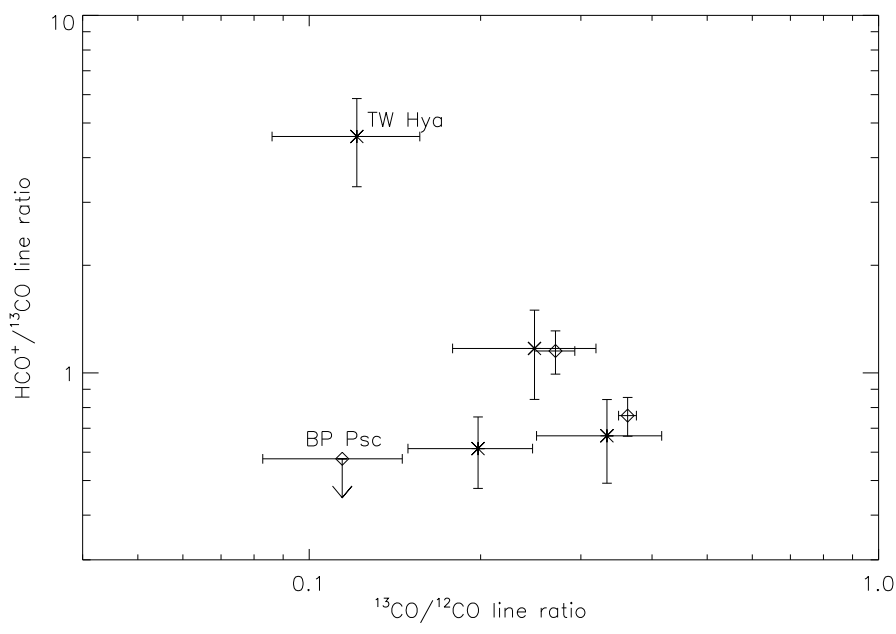

Fig. 3. $\mathrm{HCO}^{+} /{ }^{13} \mathrm{CO}$ vs. ${ }^{13} \mathrm{CO} /{ }^{12} \mathrm{CO}$ line ratios for BP Psc and for the (6) pre-MS stars measured to date (including the evolved cTTS TW Hya). Data points corresponding to JCMT $15 \mathrm{~m}$ measurements of the integrated line intensities in the $\mathrm{HCO}^{+}(4-3)$ and ${ }^{13} \mathrm{CO}(3-2)$ transitions (Thi et al. 2004) are indicated with asterisks; points corresponding to IRAM $30 \mathrm{~m}$ measurements of the integrated line intensities in the $\mathrm{HCO}^{+}(3-2)$ and ${ }^{13} \mathrm{CO}(2-1)$ transitions are indicated with diamonds (BP Psc, this paper; other stars, Dutrey et al. 1997).

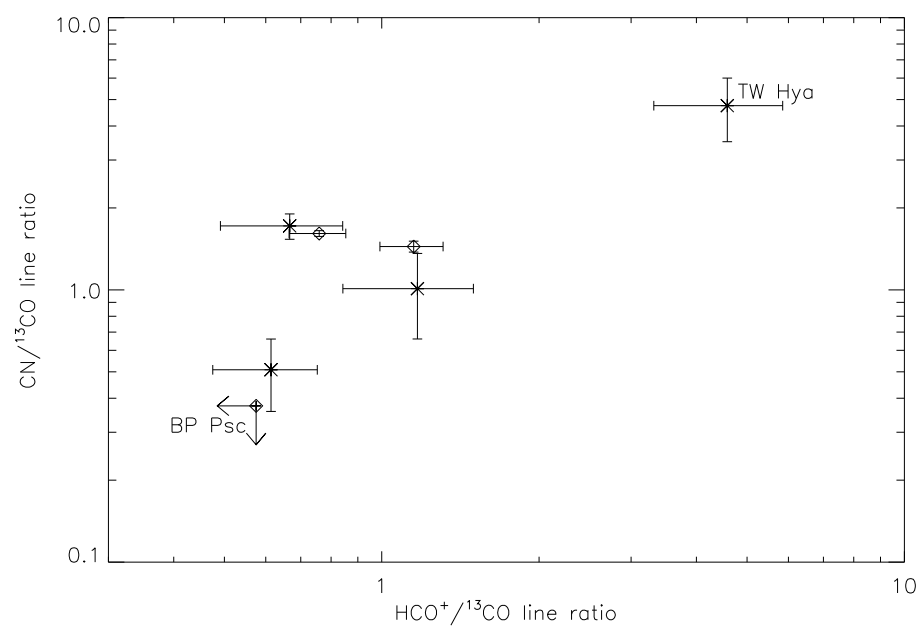

Fig. 4. $\mathrm{CN} /{ }^{13} \mathrm{CO}$ vs. $\mathrm{HCO}^{+} /{ }^{13} \mathrm{CO}$ line ratios for BP Psc and pre-MS stars. Data points corresponding to JCMT $15 \mathrm{~m}$ measurements of the integrated line intensities in the $\mathrm{HCO}^{+}(4-3), \mathrm{CN}(3-2)$ and ${ }^{13} \mathrm{CO}(3-2)$ transitions (Thi et al. 2004) are indicated with asterisks; points corresponding to IRAM $30 \mathrm{~m}$ measurements of the $\mathrm{HCO}^{+}(3-2), \mathrm{CN}(2-1)$, and ${ }^{13} \mathrm{CO}(2-1)$ transitions are indicated with diamonds (BP Psc, this paper; other stars, Dutrey et al. 1997). The lone data point near the BP Psc upper limits is the Herbig Ae star MWC 480.

sources for which line intensities have been published to date (TW Hya: Kastner et al. 1997 and Thi et al. 2004; DM Tau \& GG Tau: Dutrey et al. 1997; LkCa 15, HD 163296, and MWC 480: Thi et al. 2004). The ratios have been calculated from line intensity data obtained at transitions that lie within $\sim 15 \%$ or less in frequency, so are relatively insensitive to beam dilution effects; these and other systematic errors in the line ratios (e.g., corrections for the different relative contributions from unmeasured CN hyperfine structure lines at each rotation transition; Skatrud et al. 1983) are similar to or smaller than the typical measurement uncertainties. Assuming the ${ }^{13} \mathrm{CO}$ emission from all stars is optically thin, the ${ }^{13} \mathrm{CO} /{ }^{12} \mathrm{CO}$ line ratio should provide a measure of ${ }^{12} \mathrm{CO}$ column density (Sect. 3.2). Meanwhile, because the typical disk gas densities are sufficient to wellexcite the observed transitions (Sect. 3.2), the $\mathrm{HCO}^{+} /{ }^{13} \mathrm{CO}$ and $\mathrm{CN} /{ }^{13} \mathrm{CO}$ ratios serve as measures of the relative abundances (as opposed to the ease of excitation) of $\mathrm{HCO}^{+}$and $\mathrm{CN}$, respectively (again assuming ${ }^{13} \mathrm{CO}$ is optically thin).

In both Figs. 3 and 4, five of the six previously observed preMS stars appear clustered together. The outlier among the preMS stars is the "old" (age $\sim 8$ Myr) cTTS TW Hya. Although ${ }^{12} \mathrm{CO}$ is optically thick in all of the objects, the ${ }^{12} \mathrm{CO}$ optical depth of the TW Hya disk is smaller than that of the other preMS stars (see also Table 8 of Thi et al. 2004), likely reflecting its relatively evolved state. TW Hya also displays the largest $\mathrm{HCO}^{+} /{ }^{13} \mathrm{CO}$ and $\mathrm{CN} /{ }^{13} \mathrm{CO}$ line ratios among the pre-MS stars.

Figure 4 is indicative of a correlation between the $\mathrm{HCO}^{+} /{ }^{13} \mathrm{CO}$ and $\mathrm{CN} /{ }^{13} \mathrm{CO}$ line ratios in pre-MS circumstellar molecular disks. Furthermore, the star with the largest $\mathrm{HCO}^{+} /{ }^{13} \mathrm{CO}$ and $\mathrm{CN} /{ }^{13} \mathrm{CO}$ line ratios, TW Hya, exhibits the largest quiescent X-ray luminosity: $L_{\mathrm{X}}=1.4 \times 10^{30} \mathrm{erg} \mathrm{s}^{-1}$ (Kastner et al. 2002), compared with $L_{X} \lesssim 5 \times 10^{29} \mathrm{erg} \mathrm{s}^{-1}$ for those stars for which X-ray data have been published to date (HD 163296, Stelzer et al. 2006; DM Tau, Güdel et al. 2006; GG Tau, Stelzer \& Neuhauser 2001). TW Hya also possesses the smallest molecular disk radius and mass (Dutrey et al. 1997; Thi et al. 2004, and references therein). Hence the apparent correlation of $\mathrm{HCO}^{+} /{ }^{13} \mathrm{CO}$ and $\mathrm{CN} /{ }^{13} \mathrm{CO}$, combined with the inferred large (2-3 order of magnitude) enhancement of the $\mathrm{CN} / \mathrm{HCN}$ and $\mathrm{HCO}^{+} / \mathrm{CO}$ abundance ratios in all of the pre-MS disks, relative to values of these ratios in molecular cloud cores (Thi et al. 2004), supports the interpretation that high molecular ionization rates - most likely due to irradiation by X-rays emitted from stellar coronae and/or from star-disk interfaces - enhance the abundances of both $\mathrm{HCO}^{+}$and $\mathrm{CN}$ in these disks.

If disk ionization by central $\mathrm{X}$-ray sources is responsible for the potential correlation between $\mathrm{HCO}^{+} /{ }^{13} \mathrm{CO}$ and $\mathrm{CN} /{ }^{13} \mathrm{CO}$ apparent in Fig. 4, then this correlation indicates that the molecular gas disks lie in a regime where both $\mathrm{HCO}^{+}$and $\mathrm{CN}$ abundances are roughly proportional to X-ray ionization rate (Lepp \& Dalgarno 1996, their Figs. 2, 3). More specifically - noting that the $\mathrm{HCO}^{+} /{ }^{12} \mathrm{CO}$ and $\mathrm{CN} /{ }^{12} \mathrm{CO}$ number ratios in the pre-MS disks are inferred to be as large as $\sim 3 \times 10^{-4}$ and $\sim 2 \times 10^{-3}$, respectively (Thi et al. 2004) - the Lepp \& Dalgarno models indicate that ionization rates in the 6 previously measured pre-MS disks lie in the range $10^{-15}-10^{-13} \mathrm{~s}^{-1}$ (given a representative disk number density $n \sim 10^{7} \mathrm{~cm}^{-3}$ for the molecular line-emitting regions; Thi et al. 2004). This range is several orders of magnitude larger than the canonical molecular ionization rate due to cosmic rays.

The ${ }^{13} \mathrm{CO} /{ }^{12} \mathrm{CO}$ ratio of BP Psc is similar to that of TW Hya, and is smaller than the ratios characteristic of the (younger) preMS stars in Fig. 3. If BP Psc were pre-MS, this comparison would suggest that the BP Psc disk is as highly evolved as the disk orbiting TW Hya. However, the $\mathrm{HCO}^{+} /{ }^{13} \mathrm{CO}$ and $\mathrm{CN} /{ }^{13} \mathrm{CO}$ line ratio upper limits measured for BP Psc are at least a factor $\sim 30$ lower than those of TW Hya and a factor 3-10 lower than all but one of the other pre-MS stars. The only pre-MS star near which BP Psc may lie in Fig. 4 is the (intermediate-mass) Herbig Ae star MWC 480 - a star with which BP Psc, if a (K-type, lowmass) pre-MS star, otherwise would have little in common. If the trend observed in Fig. 4 is indeed indicative of X-ray ionization of $\mathrm{H}_{2}$, then its low $\mathrm{HCO}^{+} /{ }^{13} \mathrm{CO}$ and $\mathrm{CN} /{ }^{13} \mathrm{CO}$ ratios imply BP Psc has an anomalously low X-ray flux at its disk surface, compared with the other (pre-MS) star-disk systems (with the possible exception of MWC 480). 
Unfortunately, the only X-ray observation of BP Psc obtained thus far - a nondetection in the ROSAT All-Sky Survey (RASS) - cannot be used to test this hypothesis. The RASS nondetection (PSPC count rate $\lesssim 0.1 \mathrm{~s}^{-1}$ ) implies an intrinsic X-ray flux upper limit $F_{\mathrm{X}} \lesssim 9 \times 10^{-12} \mathrm{erg} \mathrm{s}^{-1} \mathrm{~cm}^{-2}(0.1-2.0 \mathrm{keV})$ assuming ${ }^{4} T_{\mathrm{X}}=10^{7} \mathrm{~K}$ and an intervening absorbing column $N_{\mathrm{H}}=8 \times 10^{21} \mathrm{~cm}^{-2}$ (adopting the $N_{H_{2}}$ value obtained by $\mathrm{Z} 08$ and correcting for the ${ }^{12} \mathrm{CO}$ optical depth determined in Sect. 3.1), or an X-ray luminosity $L_{\mathrm{X}} \lesssim 10^{31} \mathrm{erg} \mathrm{s}^{-1}$ for an assumed source distance of $\leq 100 \mathrm{pc}$. Hence, if BP Psc is a pre-MS star, the RASS nondetection would be consistent with a quiescent $L_{X}$ even larger than that of TW Hya $\left(1.4 \times 10^{30} \mathrm{erg} \mathrm{s}^{-1}\right.$; Kastner et al. 2002). If BP Psc is, instead, a post-MS G star at a distance $\sim 300 \mathrm{pc}$ (Z08), the RASS nondetection does not preclude the possibility that its $L_{\mathrm{X}}$ is comparable to that of the more X-ray-luminous G-type giants (Gondoin 2005).

\section{Conclusions}

The suite of molecular line data obtained thus far for BP Psc (Table 1, Fig. 1) confirms that its circumstellar disk in certain respects resembles those of pre-MS stars - but also reveals some fundamental differences. The BP Psc CO line profiles indicate Keplerian rotation of at least $\sim 0.1$ Jupiter masses of disk gas around a central star(s) whose mass lies the range 0.5-0.9 $M_{\odot}$, assuming pre-main sequence status (Sects. 3.1, 3.2 and Z08), although the profiles are also suggestive of a disk temperature gradient that is somewhat steeper than the canonical $r^{-1 / 2}$ characteristic of pre-MS star molecular disks (BS93). The ${ }^{12} \mathrm{CO}$ optical depth of the BP Psc disk (as inferred from its ${ }^{13} \mathrm{CO}(2-1) /{ }^{12} \mathrm{CO}(2-1)$ line ratio) is similar to that of the highly evolved (age $\sim 8 \mathrm{Myr}$ ) cTTS TW Hya, consistent with the interpretation that - like TW Hya - BP Psc is an isolated, "old" (yet actively accreting) TTS.

On the other hand, the $\mathrm{HCO}^{+} /{ }^{13} \mathrm{CO}$ and $\mathrm{CN} /{ }^{13} \mathrm{CO}$ line ratios of BP Psc are smaller than those of most (if not all) preMS star disks observed to date in these molecules. In particular, its disk chemistry differs sharply from that of TW Hya, its presumed closest pre-MS analog (Fig. 4). Indeed, in this key respect, the circumstellar molecular disk of BP Psc would appear to have more in common with, e.g., the expanding envelopes of yellow supergiants (which display $\mathrm{HCO}^{+}(3-2) /{ }^{13} \mathrm{CO}(2-1)$ ratios $\lesssim 10$ and $\mathrm{CN}(2-1) /{ }^{13} \mathrm{CO}(2-1)$ ratios $\lesssim 5$; Quintana-Lacaci et al. 2007) than with pre-MS disks. These results therefore are consistent with the notion that the BP Psc disk may have its origins not in the star formation process but, rather, in a catastrophic interaction with a close companion during the primary's ascent of the red giant branch (Z08). The minimum disk gas mass and angular momentum inferred under the assumption that BP Psc is a postMS star at a distance of $300 \mathrm{pc}-$ i.e., $\sim 1$ Jupiter mass (Sect. 3.2) distributed over a disk with radius $\sim 150 \mathrm{AU}-$ is consistent with such a companion-engulfing scenario (see discussion in Sect. 4.7 of Z08).

The feeble output from the BP Psc disk in the $\mathrm{HCO}^{+}$and $\mathrm{CN}$ lines further implies a low molecular ionization rate, suggesting that - in contrast to pre-MS star-disk systems - the BP Psc system lacks a strong, central X-ray source. A deep Chandra observation of BP Psc, presently scheduled for late 2008, should result in a sensitive measurement of the "hard" (1.0-10 keV)
X-ray flux incident on the BP Psc disk, providing a test of this interpretation.

However, detection of a large X-ray flux from BP Psc - while leaving open the question of the origin of its anomalous molecular emission line ratios - would shed little additional light on its evolutionary status. This is because its projected rotational velocity may be as large as $v \sin i \sim 32 \mathrm{~km} \mathrm{~s}^{-1}$ (Z08) which, given a radius typical of late-G giants $\left(\sim 10 R_{\odot}\right)$, would imply a period of only $\sim 2$ days. This period is similar both to those of rapidly rotating pre-MS stars and to those of X-ray-luminous, Gtype giants of the FK Com class (Gondoin 2005, and references therein). The approximate upper limit on the kinematic mass of BP Psc assuming a distance of 300 pc, $1.8 M_{\odot}$ (Sect. 3.1), would be consistent with FK Com status. As FK Com stars are thought to be the products of stellar mergers (Heunemoerder et al. 1993, and references therein), the comparison has interesting ramifications for the recent history of BP Psc, under the hypothesis that it is a post-MS star: if BP Psc is indeed a giant now engulfing a close companion (see Soker 1998) - forming a disk and driving jets in the process (e.g., Nordhaus \& Blackman 2006, and references therein) - we may be witnessing the "birth" of FK Com.

Acknowledgements. The authors wish to acknowledge useful comments and suggestions by David Meier, David Wilner, and the referee. J.H.K. thanks the staff of the Laboratoire d'Astrophysique de Grenoble for their support and hospitality during his yearlong sabbatical visit to that institution.

\section{References}

Backman, D. E., \& Paresce, F. 1993, in Protostars and Planets III, ed. E. H. Levy, \& J. I. Lunine, 1253

Beckwith, S., \& Sargent, A. 1993, ApJ, 402, 280 (BS93)

Deguchi, S., Izumiura, H., Kaifu, N., et al. 1990, ApJ, 351, 522

Dutrey, A., Guilloteau, S., \& Guelin, M. 1997, A\&A, 317, L55

Glassgold, A. E., Najita, J. R., \& Igea, J. 2004, ApJ, 615, 972

Glassgold, A. E., Najita, J. R., \& Igea, J. 2007, ApJ, 656, 515

Gondoin, P. 2005, A\&A, 444, 531

Guedel, M., Briggs, K. R., Arzner, K., et al. 2007, A\&A, 483, 353

Huenemoerder, D. P., Ramsey, L. W., Buzasi, D. L., \& Nations, H. L. 1993, ApJ, 404, 316

Kastner, J. H., Zuckerman, B., Forveille, T., \& Weintraub, D. A. 1997, Science, 277,67

Kastner, J. H., Vrtilek, S. D., \& Soker, N. 2001, ApJ, 550, L189

Kastner, J. H., Huenemoerder, D. P., Schulz, N., Canizares, C. R., \& Weintraub, D. A. 2002, ApJ, 567, 434

Lagrange, A.-M., Backman, D. E., \& Artymowicz, P. 2000, in Protostars and Planets IV, ed. V. Mannings, A. P. Boss, \& S. Russell, 639

Lepp, S., Dalgarno, A. 1996, A\&A, 306, L21

Nordhaus, J., \& Blackman, E. G. 2006, MNRAS, 370, 2004

Omodaka, T., Kitamura, Y., \& Kawazoe, E. 1992, ApJ, 396, L87

Quintana-Lacaci, G., Bujarrabal, V., Castro-Carrizo, A., \& Alcolea, J. 2007, A\&A, 471, 551

Skatrud, D. D., De Lucia, F. C., Blake, G. A., \& Sastry, K. V. L. N. 1983, J. Mol. Spec., 99, 35

Stephenson, C. B. 1986, ApJ, 300, 779

Stelzer, B., \& Neuhauser, R. 2001, A\&A, 377, 538

Stelzer, B., Micela, G., Hamaguchi, K., \& Schmitt, J. H. M. M. 2006, A\&A, 457, 223

Soker, N. 1998, ApJ, 496, 833

Thi, W.-F., van Zadelhoff, G.-J., \& van Dishoeck, E. F. 2004, A\&A, 425, 955

Webb, R., Zuckerman, B., Platais, I., et al. 1999, ApJ, 512, L63

Zuckerman, B. 2001, ARA\&A, 39, 549

Zuckerman, B., et al. 2008, ApJ, in press (Z08),

[arXiv: 0802.0226]

Zuckerman, B., \& Song, I. 2004, ARA\&A, 42, 685

Zuckerman, B., Forveille, T., \& Kastner, J. H. 1995, Nature, 373, 494

\footnotetext{
${ }^{4}$ The characteristic $T_{\mathrm{X}}$ of BP Psc would be lower than $10^{7} \mathrm{~K}$ if its $\mathrm{X}$-ray spectrum resembles that of TW Hya $\left(T_{\mathrm{X}} \sim 3 \times 10^{6} \mathrm{~K}\right.$; Kastner et al. 2002). If so, the RASS nondetection of BP Psc would provide even poorer constraints on its intrinsic X-ray flux $F_{\mathrm{X}}$ and, hence, $L_{\mathrm{X}}$.
} 